\title{
MySQL/PHP web database applications for IPAC proposal submission
}

\author{
Megan K. Crane, Lisa J. Storrie-Lombardi, Nancy A. Silbermann, Luisa M. Rebull \\ Spitzer Science Center, California Institute of Technology, MS 220-6 \\ 1200 E. California Blvd., Pasadena, CA, USA 91125
}

\begin{abstract}
The Infrared Processing and Analysis Center (IPAC) is NASA's multi-mission center of expertise for long-wavelength astrophysics. Proposals for various IPAC missions and programs are ingested via MySQL/PHP web database applications. Proposers use web forms to enter coversheet information and upload PDF files related to the proposal. Upon proposal submission, a unique directory is created on the webserver into which all of the uploaded files are placed. The coversheet information is converted into a PDF file using a PHP extension called FPDF. The files are concatenated into one PDF file using the command-line tool pdftk and then forwarded to the review committee. This work was performed at the California Institute of Technology under contract to the National Aeronautics and Space Administration.
\end{abstract}

Keywords: Infrared Processing and Analysis Center, Proposal submission, Web Database Application, MySQL, PHP

\section{INTRODUCTION}

The Infrared Processing and Analysis Center (IPAC), located on the campus of the California Institute of Technology, is the institutional home of the Spitzer Science Center (SSC), the Michelson Science Center (MSC), and the NASA Herschel Science Center (NHSC). Applications for the Spitzer Fellowship program and proposals for the SSC Director's Discretionary Time (DDT) program, the NHSC Theoretical Research/Laboratory Astrophysics and Key Program Data Analysis programs, NASA-Keck, and SIM Science Studies proposals are ingested and reviewed via web database applications.

A web database application is a web site that is driven from data stored in a database. The application is generally built around a three-tier architecture model. At the base is the database tier, which contains the data that users may create, delete, modify, or query. The middle tier is the application logic, which consists of the webserver and the scripts that communicate the data between the other two tiers. The top tier is the client web browser software.

Our applications have been developed on Mac OS X using the popular open source trio of the PHP server-side scripting language, the MySQL database management system, and the Apache webserver. All applications utilize the Secure Sockets Layer (SSL) protocol, which provides encryption services to ensure secure web transactions. PHP was chosen for its power, flexibility, platform and browser independence, compatibility with MySQL, and its native session and cookie management, which are used to control user access. MySQL is a high-performance backend database management system with a high-speed transactional processing system that can support over a hundred simultaneous users of the software.

\subsection{System Requirements}

1. A webserver such as Apache that can execute PHP scripts

2. PHP version 4.x or 5.x

3. MySQL version 4.x or 5.x

4. Postfix or sendmail to send emails via PHP scripts

5. FPDF - a free PHP class used to generate PDF files

6. PDF Toolkit - a command line tool used to concatenate PDF files

\subsection{Load Test}

A load test of the NHSC proposal submission system was conducted using SilkPerformer $6.6{ }^{1}$. The purpose of the test was to determine whether the system could ingest 100 proposals in one hour, and whether the system could handle 100 users simultaneously submitting proposals. The test indicated that the system could easily ingest 100 proposals in one

Observatory Operations: Strategies, Processes, and Systems II, edited by Roger J. Brissenden, David R. Silva, Proc. of SPIE Vol. 7016, 701626, (2008) - 0277-786X/08/\$18 · doi: 10.1117/12.788567 
hour, but could handle only $25-50$ users simultaneously submitting proposals. For all of the aforementioned IPAC programs, the number of expected proposals is generally less than 50 , so no major performance bottlenecks were predicted during operational use.

\subsection{Motivation for web-based proposal submission system}

In the past, most IPAC proposals and related materials (e.g. letters of recommendation, observation requests) were submitted via email to the institution that issued the call for proposals. Upon receipt of the proposal, IPAC staff would have to create a dedicated directory on hard disk to store the proposal, collate all of the related materials as they were submitted, and deliver hardcopies or electronic versions to the review committee. Acceptance/rejection letters, including the comments entered by the review committee, and data analysis funding notifications had to be sent one at a time to the principal investigator and all of the co-investigators.

A web-based proposal submission system was desired because it would automate and simplify the process of keeping track of the proposals, and would be cost effective for small numbers of proposals if it were simple to use and maintain. Directories could be automatically created to store the proposal materials, and automatic notification emails could be sent to the proposers. Spitzer Fellowship letters of recommendation could be automatically placed in the appropriate applicant's directory, instead of having to retrieve them from email attachments and place them in the directory by hand, saving IPAC staff a considerable amount of time and energy. Storing the proposal information in a database and a password-protected website would keep the information secure and easier to manage.

\subsection{IPAC projects that now use web-based proposal submission systems}

The Spitzer Science Center (SSC) (http://ssc.spitzer.caltech.edu/) supports the science operations of NASA's infrared Spitzer Space Telescope. The Spitzer Fellowship Program (http://ssc.spitzer.caltech.edu/geninfo/fellows/) provides an opportunity for highly qualified recent postdoctoral scientists to conduct independent research with the goal of enhancing the overall scientific return from the Spitzer mission. Applications for the program are solicited on an annual basis, and the SSC typically receives over 100 applications for only 5 positions.

The Spitzer Director's Discretionary Time (DDT) program (http://ssc.spitzer.caltech.edu/geninfo/ddt/) is allocated five percent of the available Spitzer observing time, and is intended to facilitate observations that address emerging scientific topics or areas missed in the proposal review process. DDT proposals can be submitted at any time during the year.

The NASA Herschel Science Center (NHSC) (http://www.ipac.caltech.edu/Herschel/nhsc.shtml) was established to provide the US astronomical community with science and observational support throughout all phases of the Herschel Space Observatory mission. The NHSC issued calls for proposals for Key Program Data Analysis (DA/KP) and Theoretical Research and Laboratory Astrophysics (TR/LA), and received $53 \mathrm{DA} / \mathrm{KP}$ proposals and $28 \mathrm{TR} / \mathrm{LA}$ proposals.

The Michelson Science Center (MSC) (http://msc.caltech.edu/) administers NASA's portion of the time on the Keck Telescopes, issuing proposal solicitation, conducting the peer review, and finalizing selections for investigations. The MSC received about 30 proposals for NASA-Keck time for the 2008A and 2008B semesters.

The MSC and the Space Interferometer Mission (SIM) (http://planetquest.jpl.nasa.gov/SIM/sim_index.cfm) issued a call for proposals for SIM Science Studies. The solicitation objective is to enhance the science return from SIM by supporting researchers to conduct concept studies that will lead to the most scientifically productive observations using SIM.

The JPL Palomar Advisory Committee (JPAC) issued a call for proposals for observing time on the Hale 200-inch telescope and the robotic 60-inch telescope system at Palomar Observatory (http://www.astro.caltech.edu/palomarnew/) for JPL/IPAC scientists only.

\subsection{Summary of the proposal submission and review process}

Proposal submission is a one-phase process, meaning that all proposal materials are submitted at one time. Only complete proposals may be submitted, and may not be updated after submission (with the exception of NHSC proposals). The general procedure consists of the following steps:

1. Users submit coversheet information and upload related PDF files via a web form.

2. Confirmation emails are sent to the user and to IPAC staff upon successful proposal submission.

3. A unique directory is created on an IPAC webserver to store the uploaded files. 
4. Coversheet information is inserted into a database.

5. Coversheet information is converted to PDF format and concatenated with the other uploaded files to create one PDF file per proposal.

6. The complete proposal is forwarded to the review committee after the proposal deadline.

7. Reviewers read, grade, rank, and write comments about the proposals.

8. IPAC staff generate and send award letters to proposers including the reviewers' comments.

\section{MYSQL DATABASE SCHEMA \& BACKUPS}

There is a separate MySQL database for each proposal submission system. Each database contains separate tables to hold the submitted proposal information as well as information needed for reviewing the proposals after the proposal deadline. There are typically two tables to hold the submitted proposal information (proposals and cois), and three tables to hold proposal review information (users, grades, and averages).

\subsection{The proposals table}

This table holds the information that proposers enter into the web form, including the proposal title, Principal Investigator contact information, science category, requested money or observing hours, abstract, etc. It also contains placeholders for the names of the primary and secondary reviewers and the final status of the proposal, which is determined during the review meeting.

\subsection{The cois table}

This table holds the names and email addresses of all of the co-investigators on the proposal that the proposer enters into the web form. The proposal submission form allows users to enter an unlimited number of co-investigators.

\subsection{The users table}

This table contains the full name, username, password, panel assignment if necessary, and status designation (reviewer, panel chair, IPAC support staff, etc.) for each user of the proposal review system. Passwords in the users table are encrypted using the MD5 algorithm in conjunction with a unique salt, a string of extra characters prepended and appended to the password.

\subsection{The grades table}

This table holds the individual grades, votes, and comments for each reviewer for each proposal. There are typically two rounds of grading: preliminary grading, which happens over a period of several weeks before the review meeting, and voting, which happens during the review meeting.

Each reviewer is able to enter comments about each proposal, but the primary reviewer assigned to each proposal is responsible for editing the final version of the comments that will be sent to the proposers.

\subsection{The averages table}

This table holds the average, standard deviation, $\mathrm{N}$ (number of grades), minimum, and maximum grade for each proposal for each round of grading (preliminary and voting). The preliminary average is calculated automatically as reviewers enter their preliminary grades, but IPAC support team members must use the software to calculate the voted averages explicitly during the review meeting. The averages table also holds the rank of each proposal.

\subsection{Database backups}

MySQL has a built-in utility for backing up databases called mysqldump, which generates a text file of SQL commands needed to rebuild the database and all of the tables from scratch. There are two dedicated webservers for these web applications: one hosts the applications and the other is used for backups. In the weeks leading up to the review meeting, the database is backed up once a day. The mysqldump command is run via a cron job, and the resulting output file is transferred (using ssh) to the backup webserver. The file is loaded into a database on the backup webserver so there will be an exact copy of the database on each webserver. During the review meeting, the database is backed up every 15 minutes, which does not affect the performance of the applications in any way. 
Likewise, all of the application PHP scripts, HTML and CSS files, and other related files (images, proposal PDFs, etc.) are copied over to the backup server daily using cron, ssh, and rsync so that there will be an exact copy of the application on both servers.

\section{PROPOSAL SUBMISSION}

\subsection{Coversheet generation}

Proposals are submitted via web forms that can be accessed from anywhere in the world. Users begin the proposal submission process by entering coversheet information into the web form (see Figure 1). This includes contact information, proposed host institutions and references for Fellowship applicants, the proposal title, science category, requested funding amount, abstract, etc. A Javascript function allows an unlimited number of co-investigators to be entered into the web form, so users must enable Javascript in their browsers. The coversheet information is validated via Javascript, inserted into the pids table in the appropriate database, and converted into a PDF file using a free PHP class called FPDF (http://www.fpdf.org/). A PHP script generates and sends a confirmation email to the user and to IPAC staff upon successful proposal submission.

\subsection{File uploads}

Users generally need to submit files along with their coversheet information. For Spitzer Fellowship applications, these include a curriculum vitae, list of major publications and all first-author papers, summary of previous and current research, and the research proposal itself. For Spitzer DDT proposals, users must submit a full set of Astronomical Observation Requests (AORs) in addition to their proposal. All of the proposal submission systems require that users upload their proposals in PDF format, which are usually generated using templates provided by the institution that issued the call for proposals.

PHP can be used to determine whether an uploaded file is indeed a PDF. The PHP superglobal array $\$$ FILES contains all of the uploaded file information, including its original name, mime type, and size. Generally the mime type of a PDF file is identified by PHP as 'application/pdf' or 'document/pdf', depending on the user's browser and operating system. However, this process is not always reliable (see Section 5).

PHP is also used to set the maximum size of a file that can be uploaded. A hidden form element called MAX_FILE_SIZE determines the maximum file size that the browser will allow, but this value must be reinforced on the server size using the PHP directive upload_max_filesize. This value is generally set to 10-50 MB.

Upon proposal submission, a PHP script checks that the files were successfully uploaded, and creates a directory on the web server that contains the name of the applicant/proposer and the unique identifier of the proposal in the database. All of the uploaded files are moved to this directory, as well as the newly generated PDF coversheet.

\subsection{Concatenating PDF files}

The command line tool PDF Toolkit (http://www.accesspdf.com/pdftk/) is used to concatenate all of the PDF files into one PDF file per proposal. The pdftk command can be executed from within a PHP script using the PHP system() function. All of the files must truly be PDFs or the command will fail. Since the command does not include any user input, i.e. potentially tainted data, it does not constitute a command injection vulnerability. 


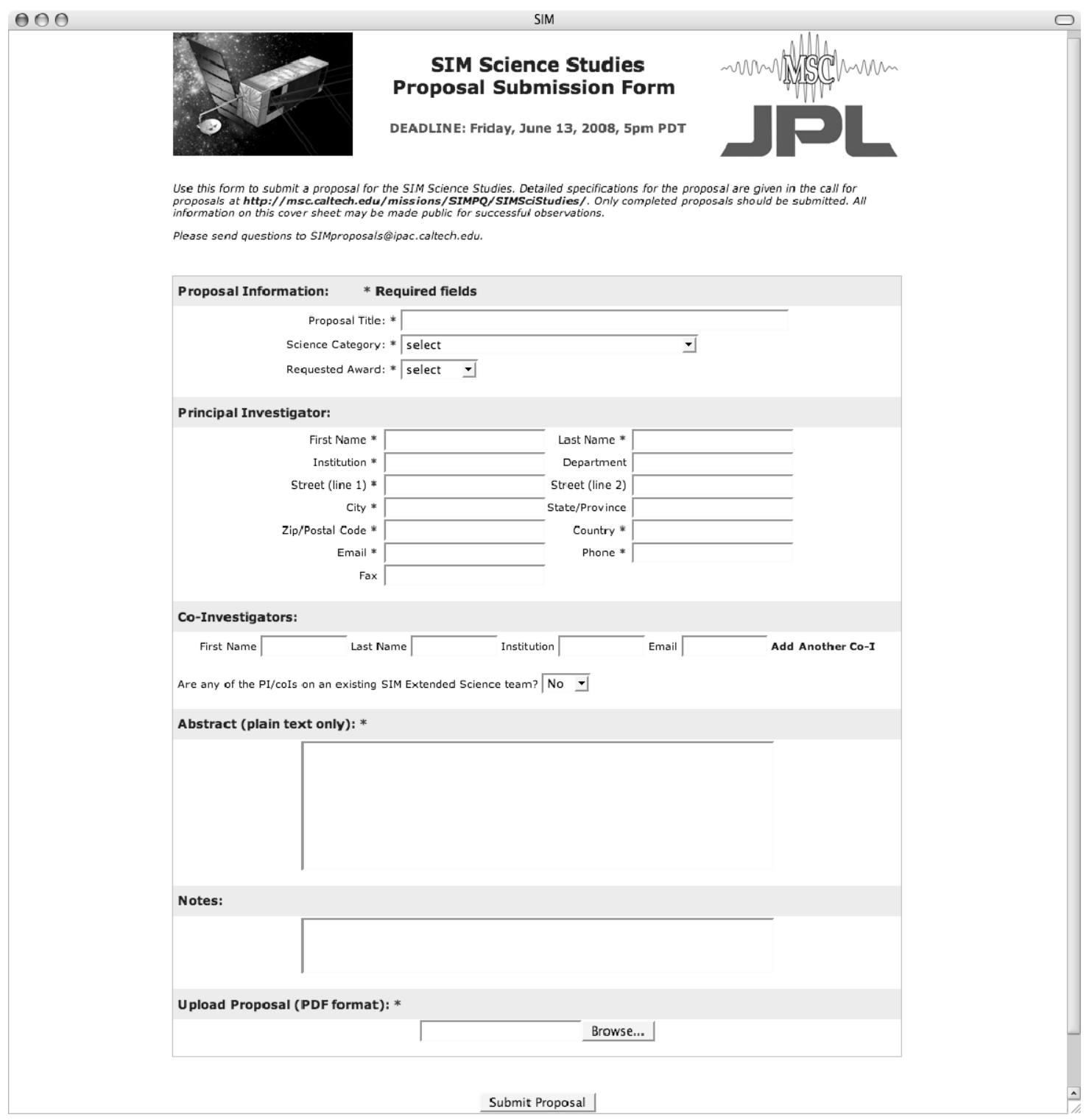

Fig. 1. SIM Science Studies proposal submission form.

\subsection{Letters of recommendation}

Spitzer Fellowship applicants are required to have three letters of recommendation and a letter of endorsement from their first choice host institution submitted in support of their application. In the past, letters were sent via email to the SSC and would often arrive before the applications were submitted. A considerable amount of time and effort were involved in placing the letters in the correct applicant's directory after the application was submitted.

A new process will be adopted for the 2009 Spitzer Fellowship applications. Referees will submit their letters electronically via a web form. They will enter the name and email address of the applicant as well as their own name and email address, and upload a PDF version of the letter (see Figure 2). The applicant will be automatically notified via email that the letter has been submitted on their behalf.

The letters will be uploaded into a directory separate from the applicants' individual directories that are created upon their application submission. SSC Community Affairs Team members will monitor the Fellowship review website and view the submitted applications alongside the submitted letters. They will be able to match up the submitted letters with the appropriate applicant, and the letters will be automatically moved to the applicant's directory. 

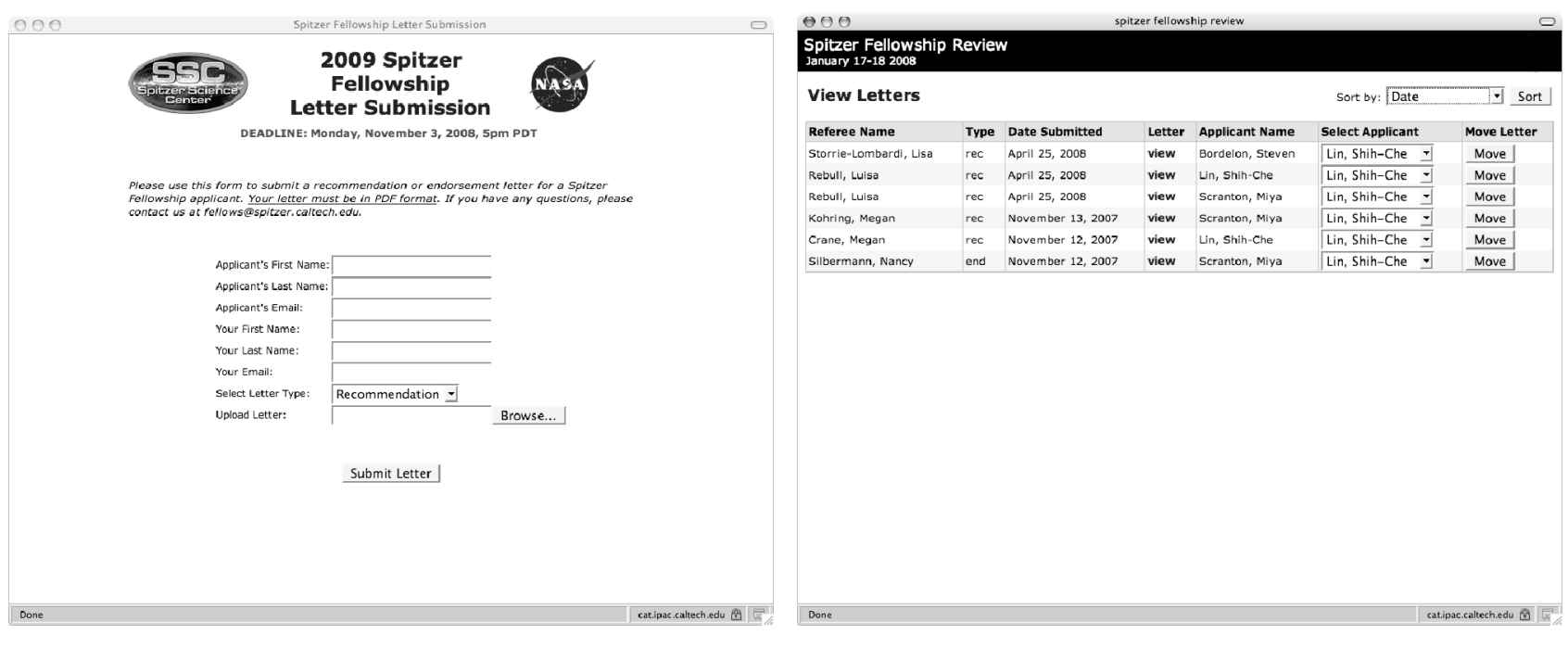

Fig. 2. Left: Spitzer Fellowship letters of recommendation/endorsement submission form. Right: viewing the submitted applications alongside the submitted letters.

\subsection{Updating submitted proposals}

For NHSC proposals, the NHSC requested that users be allowed to update their proposal if needed after submission. Upon successful proposal submission, users receive confirmation that their proposal was submitted, along with a username and password that they can use to log in to a secure website to update their proposal. There they have the ability to edit their coversheet information as well as upload replacement PDF files up until the proposal deadline, when their password will be deactivated.

IPAC support staff use a password-protected website to monitor the proposal submission process and update proposals if necessary after they have been submitted. For example, upon examination of their confirmation email, users may realize that they incorrectly entered some of their coversheet information, so they can notify IPAC staff of their mistake, and IPAC staff can update the information in the database by hand.

\subsection{Proposal submission deadline}

Proposal submission deadlines are generally at 5:00 PM PST/PDT, with an allowed grace period of 10-15 minutes. To shut off the proposal submission system, the part of the web form where the coversheet information is entered must be commented out, and a warning message displayed on the page saying that the proposal deadline has passed. This requires that someone needs to be constantly monitoring the proposal submission system up until the deadline. Alternatively, PHP or Javascript functions could be used to determine the current date and time, and only display the web form if the current date is before the proposal deadline.

\section{PROPOSAL REVIEW}

The proposal review processes generally consists of two stages: preliminary grading, which happens before the review meeting, and voting, which happens during the review meeting. The only exception is the Spitzer DDT proposals, which can be submitted and reviewed at any time.

\subsection{Logins and logouts}

When users logs in to a proposal review site, they enter a username and password into a web form. The password is encrypted using the MD5 algorithm and a salt specific to the site. If the entered password matches the password in the database for that username, login is successful. If not, the user is redirected to the login page with an error message stating that the login was unsuccessful.

Upon successful login, the appropriate users table is queried for other information about the user such as his/her status designation and the panel to which he/she is assigned. This information is registered in PHP session variables, stored in the PHP superglobal array $\$ \_S E S S I O N$, and stored in a cookie in the user's browser. This means that users must 
configure their browser to accept cookies. The session information is maintained as long as the user is logged in. Depending on their status, users are allowed access only to certain pages. For example, only the panel chairs are allowed to view the individual grades of each reviewer in their panel. Error messages are displayed and logged if a user tries to access an unauthorized page.

When a user logs out, their session information is destroyed and they are redirected back to the login page. They cannot access any pages without logging in again.

\subsection{Preliminary grades and triage}

All of the proposal review processes (except for the Spitzer DDT program) require that reviewers enter preliminary grades several days before they meet in person to discuss and re-vote on the proposals. Reviewers are required to enter a preliminary grade for each proposal, which they are able to access electronically from a password-protected review website (see Figure 3). Reviewers enter preliminary grades to one decimal place using the full scale: 1(lowest) -5 (highest), and enter 0 if they are conflicted with the proposal. Their individual average grade and standard deviation are calculated automatically as they enter their grades. They may also enter comments about the proposals, which will be combined with the comments entered by the other reviewers. Reviewers can save their grades, logout, and log back in at any time to update their grades until the preliminary grades deadline.

If there is a large number of proposals, a triage process may be implemented before the review meeting occurs. Typically the bottom third of the proposals will be triaged, leaving the panel with a more manageable number to discuss during their meeting. The panels briefly review the triaged proposals at the beginning of their meeting, particularly if there are any that have a large standard deviation in the preliminary average grade, and may elect to pull any out of triage and discuss them during their meeting.

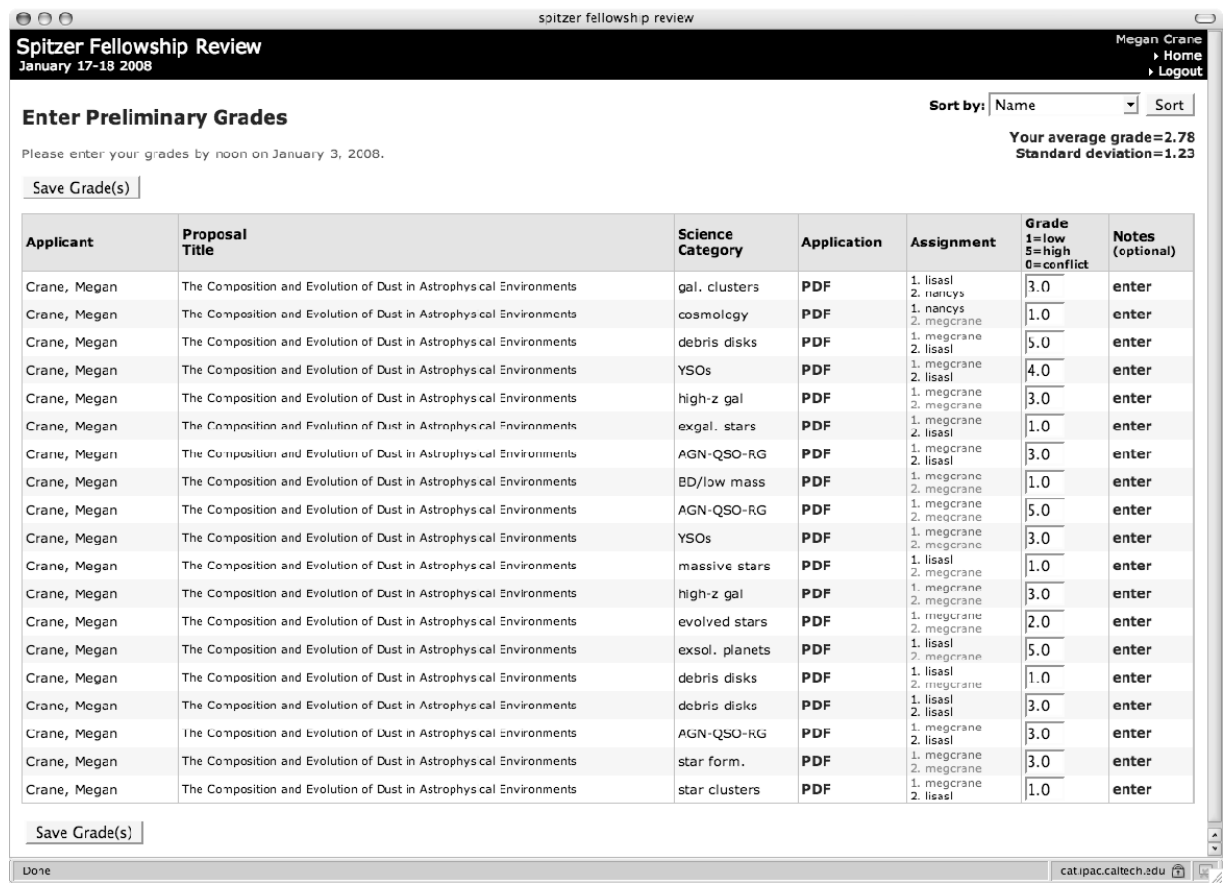

Fig. 3. Reviewers' preliminary grading page for Spitzer Fellowship applications.

\subsection{Voting and ranking}

Review meetings may be conducted in person or via teleconference, and may last anywhere from one day to a full week. The purpose of the review meeting is for the reviewers to discuss and vote on the proposals a second time, using the same scale as the preliminary grades. IPAC staff members monitor the voting process and generate a final ranked list (see Figure 4) of all of the proposals based on the panel's recommendations. The primary reviewer of each proposal is responsible for editing the final version of the comments that will be sent to the proposers. 


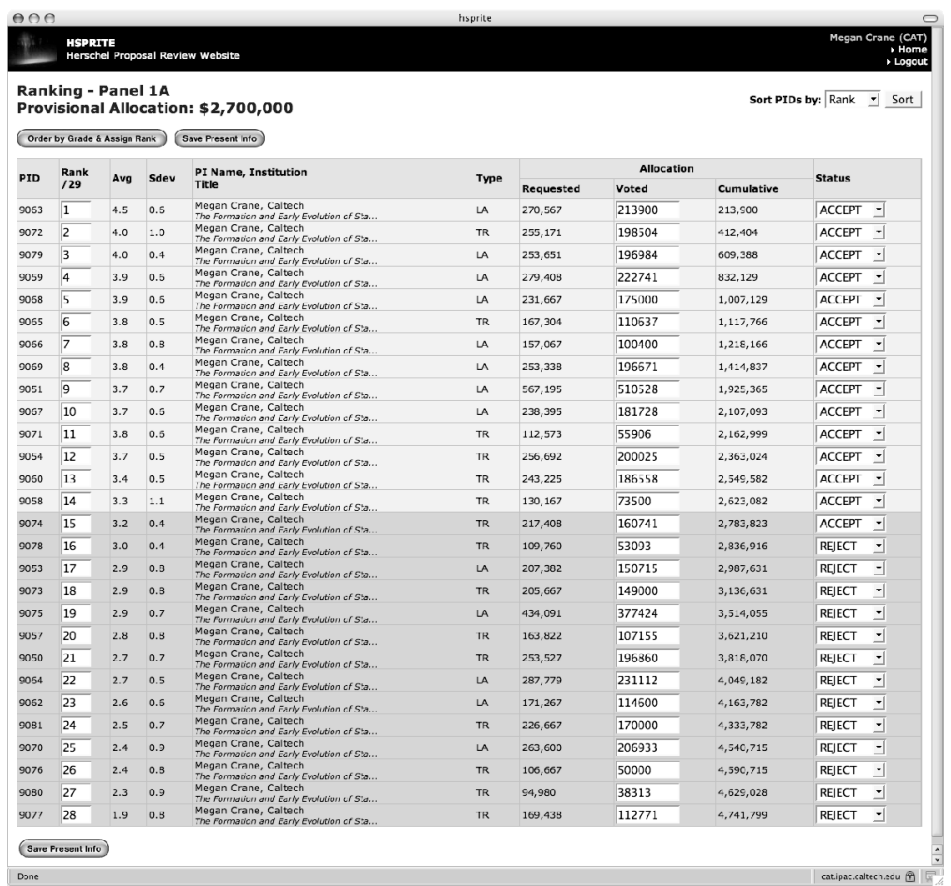

Fig. 4. NHSC TR/LA proposal ranking page.

\subsection{Award letters}

The proposal review software is used to send acceptance and rejection letters to the Principal Investigator and coinvestigators of all of the proposals after the review meeting, utilizing the PHP mail() function. The comments entered by the reviewers must be approved and edited if necessary before being sent to the proposers (see Figure 5). A form letter is generated depending on the status of the proposal (accepted or rejected), and can be sent one at a time or in batches. The PHP sleep() function is used to impose a 5-second delay between the delivery of letters so that they do not pile up in the IPAC mail queue.

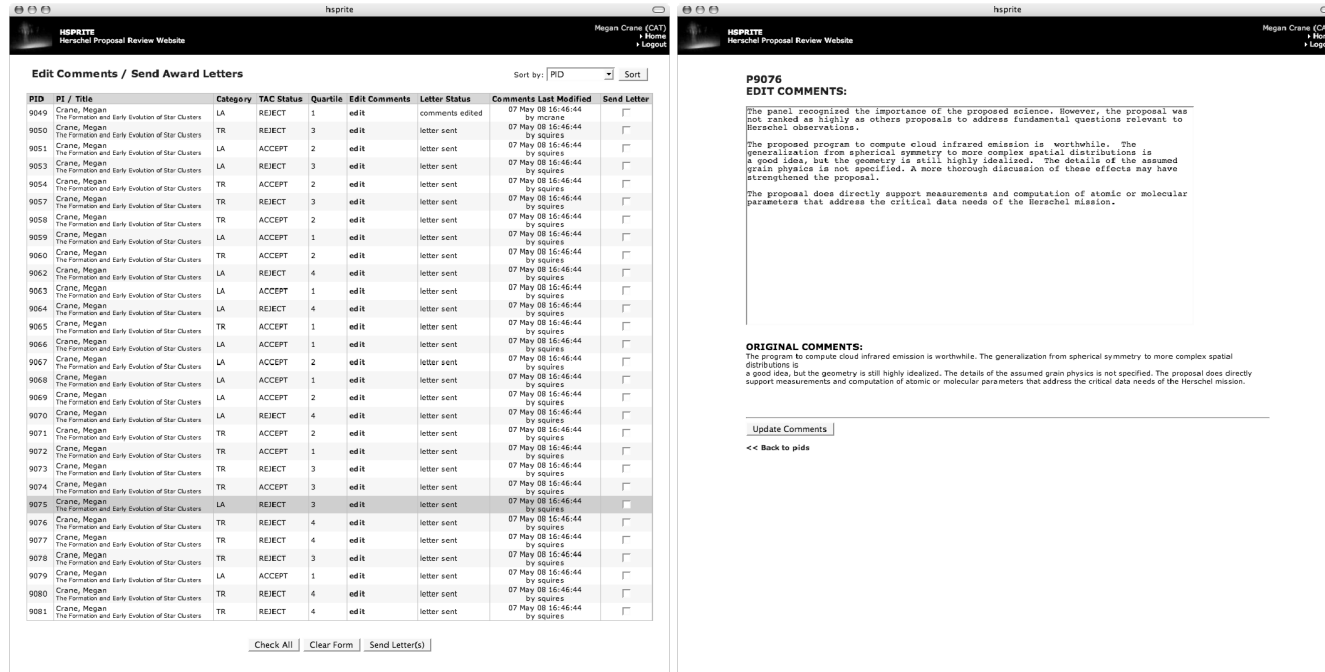

Fig. 5. Editing reviewer comments and sending award letters. 


\section{LESSONS LEARNED}

The web-based proposal submission systems have been well received by users. They can be accessed from anywhere in the world, and users don't have to download and install special software to submit their proposals. Occasionally users would encounter errors if they tried to upload a file that was larger than the maximum size allowed, but the software generally works for all browsers and operating systems.

It was found that PHP does not have a truly reliable method for determining whether a PDF file is indeed a PDF. Generally the mime type of a PDF file is identified as 'application/pdf' or 'document/pdf', depending on the user's browser and operating system. There has been at least one instance of a PDF file being identified as 'application/forcedownload', which caused the file upload to fail. The cause of this is still unknown, so PHP is no longer used to check that a file is indeed a PDF. Most users do upload PDFs, so the removal of the check has not caused any problems.

A few bugs were encountered that were related to the creation of the directories in which proposal materials are stored. A directory is created on hard disk when a proposal is submitted that is named by combining the name of the principal investigator and the unique identifier of the proposal in the database. Since the name of a directory cannot contain any characters such as spaces or apostrophes, these characters must be removed from the principal investigator's name before the directory can be successfully created. They can still exist in the database, but cannot be contained in the name of the directory.

PHP session management includes a built-in method for "garbage collection" which means that it will destroy a user's session if the user is idle for a specified amount of time. The default PHP value is 1440 seconds, or 24 minutes. Since the time that the reviewers spend discussing proposals before entering a vote often lasts longer than 24 minutes, this value had to be increased to 3 hours to ensure that users did not get unintentionally logged out. This was achieved by setting the PHP directive session.gc_maxlifetime to 10800 and rebooting the webserver.

A caveat of the one-phase proposal submission process is that all of the coversheet information has to be entered at one time. If there is a large number of co-investigators on the proposal, and there is some problem with the submission, users may be at risk of losing all of the information they just entered. For large proposals it may be beneficial to add a function that will save the coversheet information to a file on the user's desktop, so that users do not have to re-type all of the information if it gets lost.

\section{REFERENCES}

[1] Legassie, M. S., Bennett, L., Comeau, S. B., and Dodd, S. R., “Advanced load-testing techniques for a science archive", in Observatory Operations: Strategies, Processes, and Systems II, edited by Roger J. Brissenden, David R. Silva, Proceedings of SPIE Vol. 7016 (SPIE, Bellingham, WA, 2008), In Press. 